\title{
Motor functions but not learning and memory are impaired upon repeated exposure to sub-lethal doses of methyl parathion
}

\author{
Ting-Ting Sun ${ }^{1,2}$, Ian A. Paul ${ }^{1} \&$ Ing K. Ho ${ }^{1, *}$ \\ ${ }^{1}$ Department of Pharmacology and Toxicology, University of Mississippi Medical Center, Jackson, Mississippi \\ 39216, USA; ${ }^{2}$ MassGeneral Institute for Neurodegenerative Disease, Massachusetts General Hospital, \\ Harvard Medical School, Charlestown, MA 02129, USA
}

Received 2 December 2005; accepted 26 January 2006

() 2006 National Science Council, Taipei

Key words: learning and memory, methyl parathion, motor functions, repeated treatment

\begin{abstract}
Summary
Our previous work showed that repeated exposure to methyl parathion (MP) caused a prolonged inhibition of acetylcholinesterase (AChE) activity ( $~ 80 \%)$ and down-regulation of $\mathrm{M}_{1}$ and $\mathrm{M}_{2}$ muscarinic receptors (up to $38 \%$ ) in rats at brain regions, including frontal cortex, striatum, hippocampus and thalamus. In the present neurobehavioral study, we found this repeated MP treatment had suppressant effects on rat's locomotor activity. However, we observed no evidence of long-term effects of MP on associative learning and memory. Our data demonstrated that repeated exposure to MP caused some functional deficits in CNS, but motor activity and associative learning/memory process might differ in the sensitivity to its toxic effect. The motor dysfunctions in MP-treated rats may be mediated via reciprocal balance between cholinergic and dopaminergic systems at striatum following cholinergic over-stimulation. Our findings also suggest that the CNS deficits induced by repeated exposure to MP or other organophosphate (OP) pesticides cannot be attributed entirely to the inhibition of AChE. To accurately assess the neuro-toxic risk by occupational exposure to sub-lethal doses of MP, novel biomarkers besides in vivo anticholinesterase potency are needed.
\end{abstract}

\section{Introduction}

The acute toxic effects of organophosphate (OP) pesticides are associated with the inhibition of acetylcholinesterase (AChE). The toxic signs include salivation, lacrimation, excessive urination, localized fasciculation and tremor [1]. Repeated exposure of mammals to $\mathrm{OP}$ anticholinesterase (antiChE) has been reported to lead to tolerance to the behavioral effects of cholinergic over-stimulation [2]. By far the most common and consistent finding among the reports on chronic OP toxicants has been a reduction in the number of muscarinic receptors without changes in affinity [3]. Although the recep-

*To whom correspondence should be addressed. Fax: + 1-601984-1637; E-mail: iho@pharmacology.umsmed.edu tor adaptation mechanism could protect against some behavioral symptoms, the clinical symptoms seen in workers chronically exposed to OP antiChE lead us to suspect the loss of cholinergic muscarinic receptors in the brain may compromise brain function. Those workers complain of a variety of psychiatric sequelae including depression, apathy, irritability, and schizophrenia. Loss of concentration, difficulty in thinking, and especially memory impairment were also reported in clinical cases [4, 5]. Epidemiological studies provided evidence for a link between OP pesticides to Parkinson's disease [6]. Moreover, it has been shown that AChE inhibitors can induce Parkinson's-like motor dysfunction; and that parkinsonian symptoms can be exacerbated by cholinergic stimulation $[7,8]$. 
Methyl parathion (MP) is a restricted organophosphorus compound that can only be used as pesticide on agriculture crops due to its high toxicity [9]. Interest in MP as a public health concern has increased with the recognition that it was used in private residences and businesses throughout the south and central United States for a period of time during last decade [10]. By far, there are only very limited data available to assess the possible health impact of exposure to MP that could occur as a result of illegal domestic spraying or occupational exposure.

It is clear that acute exposure to high level of MP is toxic, and at extreme doses, it even leads to death; however, it is not clear that if long-term exposure to sub-lethal doses, which is a more common occurrence, is detrimental to human health. The studies on pharmacokinetics of MP through different routes of exposure suggested dermal exposure, which is the mode of exposure most likely to occur in domestic setting, increases the potential for long-term, adverse health effects [11]. In our previous study, we mimicked dermal exposure condition in a rat model. Repeated subcutaneous injections of MP to rat at $3 \mathrm{mg} / \mathrm{kg} /$ day ( $5 \%$ of dermal $\mathrm{LD}_{50}=67 \mathrm{mg} / \mathrm{kg} /$ day) for 3 weeks caused a prolonged inhibition of AChE activity in the whole blood (70\%) and brain (80-90\%), and down-regulation of muscarinic receptors in brain regions, such as striatum, hippocampus and frontal cortex [12].

In view of these findings, we suspected that consequent adverse effects in the central nervous system would be evident. For this purpose, a series of behavioral tests were conducted during and after the same MP exposure regimen. Our data indicated that repeated exposure to MP had suppressant effects on locomotor activities. However, we observed no evidence of long-term effects of MP on associative learning and memory. These findings suggest that the CNS deficits induced by repeated exposure to MP or other OP pesticides cannot be attributed entirely to the inhibition of $\mathrm{AChE}$ and reduction of cholinergic muscarinic receptors. The highly acute toxic OP pesticide does not necessarily cause severe adverse effects in a long-term exposure at sub-lethal doses. For the risk assessment of chronic neurotoxicity of OP pesticides, novel biomarkers correlating with brain functional behaviors are needed.

\section{Materials and methods}

Animals and MP exposure

Male Sprague-Dawley rats (initial body weight of 175-200 g, Harlan Inc., Indianapolis, IN) were maintained under a 12-h light/dark cycle in a temperature-controlled environment, with free access to food and water. They were housed three in one cage for at least 1 week prior to dosing. All procedures were approved by the Institutional Animal Care and Use Committee (IACUC) at the University of Mississippi Medical Center.

Rats were given s.c. injections of either MP (3 mg/kg, $\geq 99 \%$, Chem Services Inc., Westchester, PA) or vehicle (peanut oil, $1 \mathrm{ml} / \mathrm{kg}$, s.c.) for 21 days. Two hundred grams of food and $450 \mathrm{ml}$ of tap water were available for each cage every day. The body weight of each rat and food as well as water consumption per cage was recorded daily. The mean body weight change comparing to day 0 was obtained for all rats in a given group on each measuring day.

Motor activity test and learning/memory test were conducted on separate groups of rats to reduce the interaction between tests. In groups 1 and 2 ( $n=9$ for control and MP-treated), motor activities were assessed at day 0 (pre-test), and at days $2,4,7,14$ and 21 between 10 and $11 \mathrm{AM}$ before the next dosing. In groups 3 and $4(n=12$ for each), rats started to be trained in active avoidance response 2 days after the final injection. Retention of this task was retested 10 and 30 days after the completion of acquisition trials.

\section{Motor activity}

Experimental rats were transferred to the behavioral testing room and allowed for at least $1 \mathrm{~h}$ to a dim light environment. At the beginning of the test sessions, rats were placed in the center of the $44 \times 44 \mathrm{~cm}$ Plexiglas locomotor activity testing chambers separately. Rats' activities were monitored by an infrared beam monitoring system (Opto-Varimex, Columbus Instruments, $\mathrm{OH}$ ), which was linked to a PC compatible computer. The system employs detectors at body height $(4.5 \mathrm{~cm})$ and at rearing height $(12 \mathrm{~cm})$. Each detector consists of 15 sensors in the $X$ - and $Y$ coordinate planes spaced $2.54 \mathrm{~cm}$ apart with a 
maximum resolution of $1.27 \mathrm{~cm}$. This system records total distance traveled, total ambulation time, total resting time, and total time spent in stereotypy movements as well as vertical beam breaks (rearing) over $30 \mathrm{~min}$. Ambulatory time was defined as the time animals spent in ambulation e.g. forward, backward or turning movement. Vertical time was defined as the time animals stood, jumped or raised head. In addition, total distance was the accumulation of all interruptions of beams multiplied by the distance between two beams. Testing chambers were cleaned with $70 \%$ ethanol solution between subjects.

\section{Active avoidance conditioning}

Avoidance training was conducted in a metal shuttle box equipped with wire grid floors for administering shock (Columbus Instruments, Columbus, OH). The shuttle box had two chambers $(22.5 \times 24 \mathrm{~cm})$, separated by a metal barrier with an open doorway. A small incandescent light bulb was mounted on the top panel in each chamber. A speaker and sound generator were mounted above the barrier. During the experiment, the light and a $2.8 \mathrm{kHz}$ tone signaled the onset of shock. Shock intensity was set at 0.7$0.8 \mathrm{~mA}$. Infrared beams on both sides of the barrier registered crosses between chambers. Stimulus programming, shock delivery, and data recording were controlled by computer.

Two days after the final injection of MP or vehicle, acquisition training began. Twelve rats in each treatment condition were assigned to learn the active avoidance task. They were trained until they reached the criterion or, if they failed to reach the criterion, until they received 235 training trials. Rats were transferred to the testing room $1 \mathrm{~h}$ before each experimental session. Each rat received 20 trials per day (except 15 trials in the first training day). At the start of each trial, the tone and light in the shuttle box were switched on. If the rat crossed to the other chamber within $10 \mathrm{~s}$, the light and tone were switched off and a $20 \mathrm{~s}$ intertrial interval began (Avoidance). If the rat did not cross the doorway to the other chamber within $10 \mathrm{~s}$ (Avoidance), shock was delivered either until it either crossed (Escape) or for $20 \mathrm{~s}$ (Failure). All trials were followed by a $20 \mathrm{~s}$ intertrial interval. After 20 trials, the rat was removed and returned to the home cage. Analysis of acquisition was based on the percentage of trials in which animals made avoidance responses on each training day. Animals were considered to acquire active avoidance response if the avoidance reached to $80 \%$ or more. Only rats, which acquired the task, were retested on retention trials.

Retention trials were identical to training trials, except that shock was not delivered and only 10 trials were conducted on each day. Retention was tested 10 and 30 days after acquisition.

\section{Data analysis}

All data were analyzed using SPSS on a personal computer by analysis of variance (ANOVA) for repeated measurement. In addition, a series of planned comparisons between cells of the repeated measures ANOVAs were conducted with unvaried $F$-tests. Data were considered significant if $p<0.05$.

\section{Results}

\section{General toxicity}

Rats treated with MP showed general signs of OP antiAChE toxicity (e.g. tremors, purposeless chewing, and irritability). MP-induced toxicity was also resulted in a retarded growth rate observed after 4 days of MP administration (Figure 1A). There was a significant daily body weight gain in both groups $(F(19,304)=569.569, p<0.001)$, but the MP-treated rats grew consistently slower than the control rats $(F(19,304)=12.662, p<0.001)$. During the 21 days of treatment, rats receiving MP injections gained on average $30 \mathrm{~g}$ less than rat receiving only vehicle. However, daily water and food intake were not different between the groups (Figure 1B). Weight loss in rats could be due to the cholinergic over-stimulation effect of methyl parathion at peripheral nervous system, causing an increased glandular secretion and gastrointestinal tract mobility.

\section{Motor functions}

In all activities tested (distance traveled, ambulation time, vertical activity, stereotypy movement), there were significant interactions between treatment and day of administration (Figure 2). There 

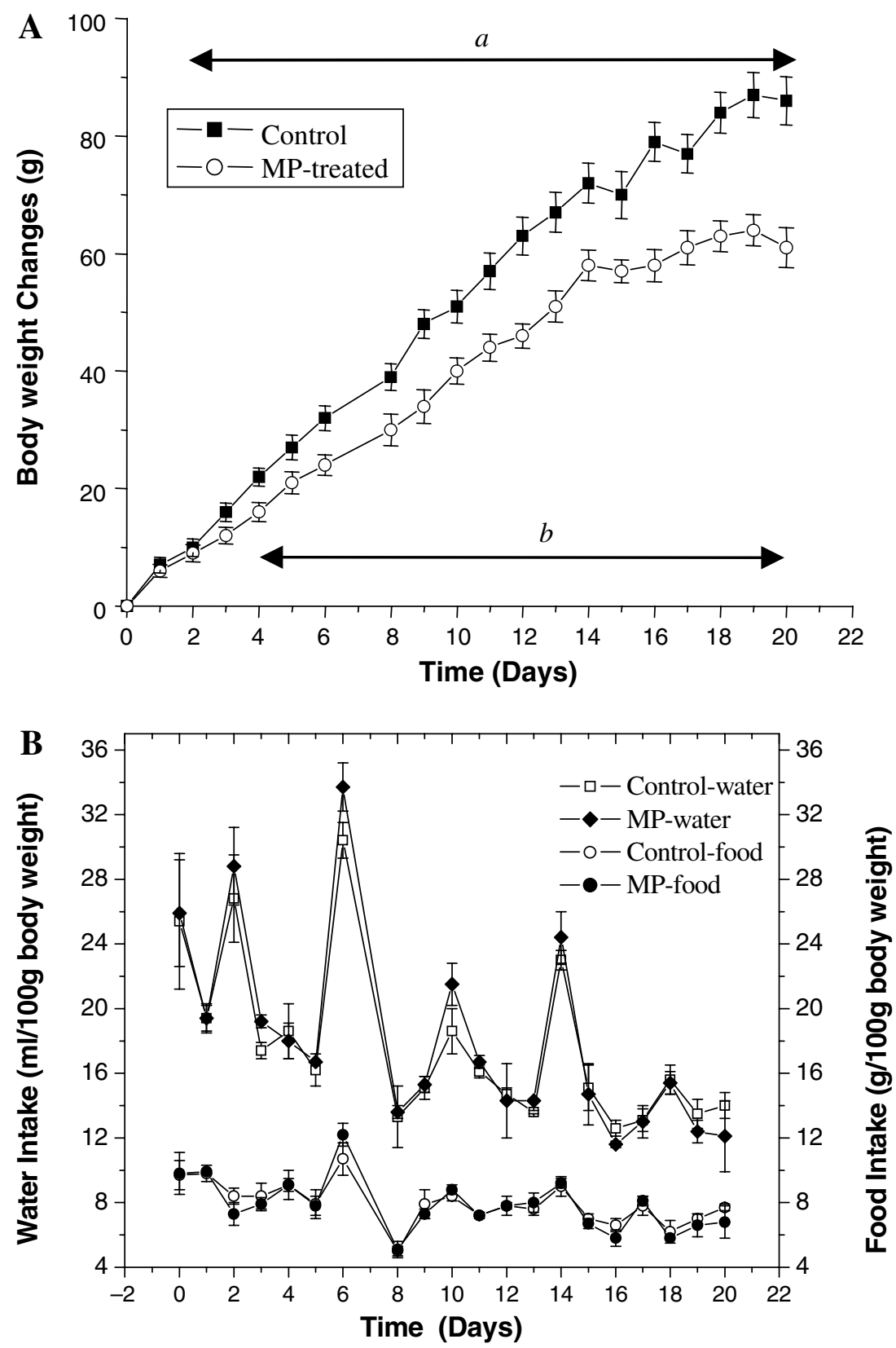

Figure 1. (A) Changes in body weight and (B) water intake ( $\mathrm{ml} / 100 \mathrm{~g}$ body weight) and food intake $(\mathrm{g} / 100 \mathrm{~g}$ body weight) during 21 days of exposure to MP $(3 \mathrm{mg} / \mathrm{kg} / \mathrm{day}, n=9)$ or vehicle $(n=9)$. Values are expressed as mean \pm SEM. $a=p<0.05$ vs. day 0 ; $b=p<0.05$ vs. control.

was no significant effect of trials on distance traveled and ambulation time within groups, however, there was a significant interaction between treatment groups and trials $(F(4,56)=3.796 ; F(4$, $56)=3.581, \quad p<0.05)$. Meanwhile, the vertical activity significantly increased with trials in both groups $(F(4,56)=32.329, p<0.001)$ and there was an interaction between groups and trials also $(F(4$, $56)=4.935, p<0.005)$. There was marginal effect of trials on stereotypy time in both groups $(F(4$, 56) $=0.850, p=0.05)$ and trial $\times$ group interaction $(F(4,56)=2.490, p=0.05)$. Planned comparison revealed that these effects were most readily apparent in the last two observations (Days 14 and 21). 

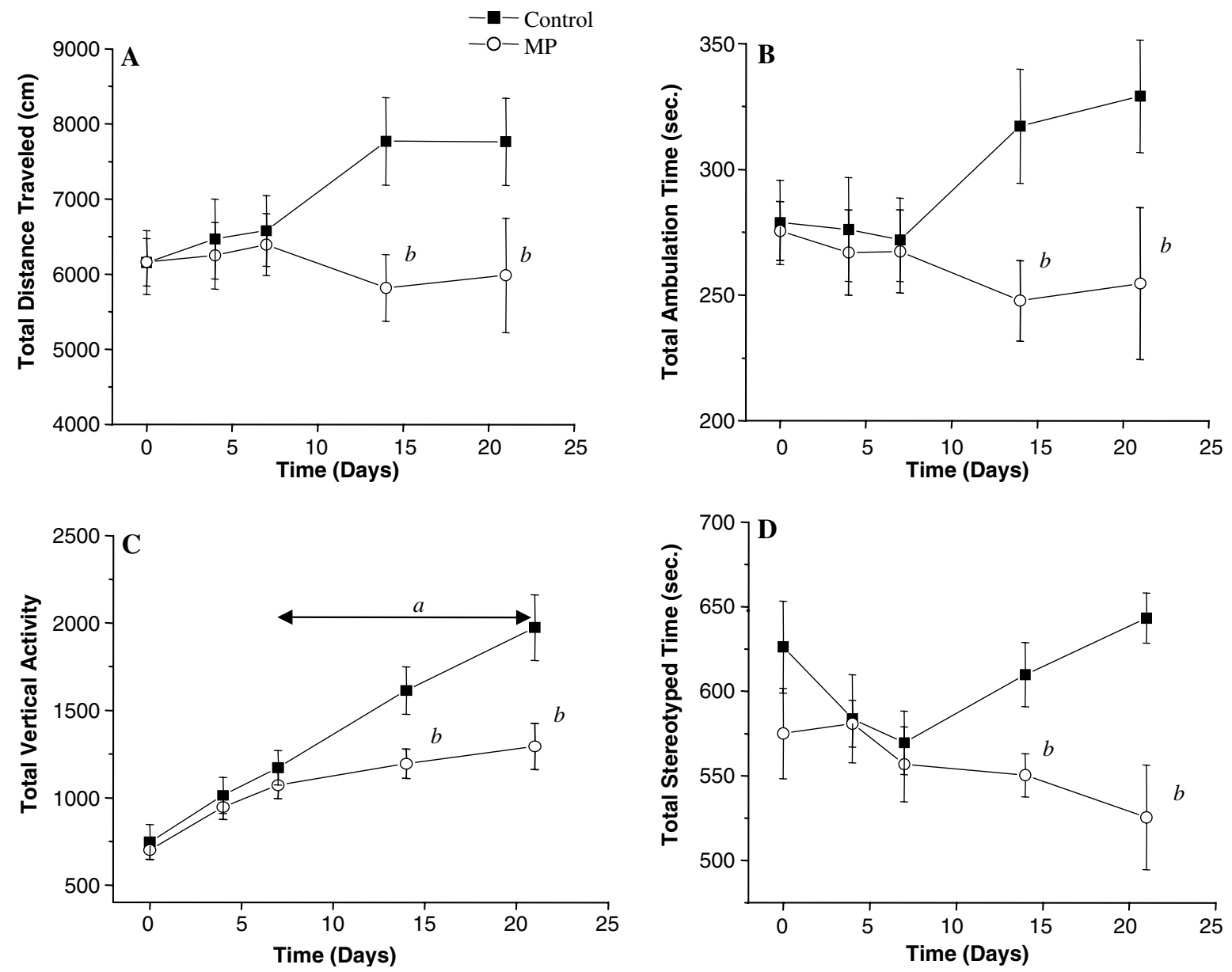

Figure 2. Total locomotor activities recorded within a period of $30 \mathrm{~min}$ (A) distance traveled; (B) ambulation time; (C) vertical activity; and (D) stereotypy movement of rats during exposure to vehicle $(n=9)$ or MP $(3 \mathrm{mg} / \mathrm{kg} / \mathrm{day}, n=9)$ for 21 days. Values are expressed as mean \pm SEM. $a=p<0.05$, vs. day $0 ; b=p<0.05$ vs. control.

Neuromuscular coordination of rats in both groups was also investigated by the rota-rod test (data not shown). Although there was significant variation between subjects, MP-treated rats displayed a tendency of poorer performance compared with the pre-treatment trial.

\section{Active avoidance acquisition and retention}

The acquisition training of active avoidance began 2 days after the final injection of MP. The number of avoidance responses made by control and MPtreated rats improved gradually over the 12 training sessions $(F(11,242)=12.862, p<0.001)$ (Figure 3A). Similarly, latency to cross diminished in both groups during the training period (Figure 3B). There was no significant interaction between groups and trials. At the end of training session, comparable proportions of rats in either group (control=8/12; $\mathrm{MP}=7 / 12$ ) acquired the active avoidance task ( $\geq 80 \%$ avoidance). Only the rats who reached the criterion during the training session were retested on the retention of the task at 10 and 30 days after the acquisition day. In the retention test, all animals, in both groups, retained the task with $100 \%$ accuracy at either time point (Figure 4).

\section{Discussion}

In our study, we used a repeated exposure animal model mimicking human exposure conditions to assess the neurological impacts of MP at sub-lethal 

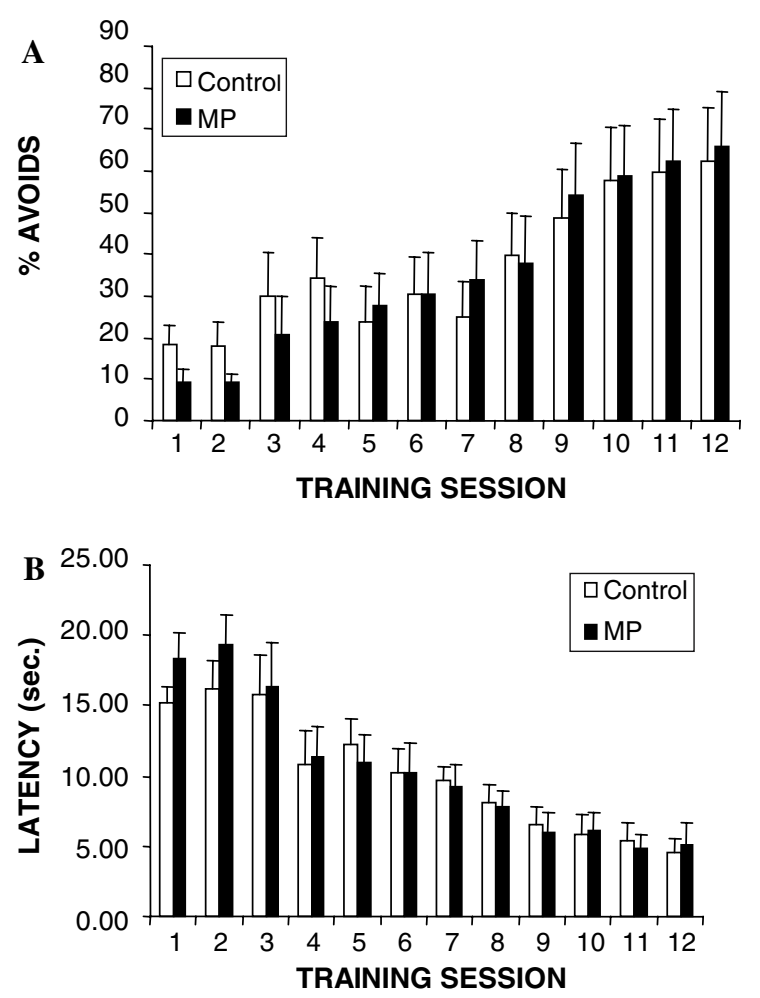

Figure 3. (A) Percentage of subjects making avoidance response per training session for 12 consecutive sessions; (B) latency of made by subjects during each training session in either control group $(n=12)$ or MP-withdrawal group $(n=12)$. Each bar represents the mean of all subjects within one group across 15-20 trials on each training day. Details for the test were described in 'Materials and methods'.

doses. Previously, we reported this treatment caused persistent AChE inhibition (about $80 \%$ ) and substantial reductions (up to $38 \%$ ) in $\mathrm{M}_{1}$ and $\mathrm{M}_{2}$ muscarinic receptor binding in brain regions (Table 1) [12]. The MP-treated rats showed typical signs of OP toxicity, such as tremor, purposeless chewing, and irritating. MP-induced toxicity was also reflected in a retard growth even without changes in drinking and eating behavior. In the parallel neurobehavioral studies, we detected substantial reduction at several aspects of motor activities, such as the distance traveled and vertical activity. Rats dosed daily with MP developed deficits in motor functions 1 week later than the observation of the significant inhibition of AChE activity and the down-regulation of muscarinic receptors in the brain. It suggests that behavioral changes in motor functions may be a consequence of the earlier neurochemical changes. The contin-

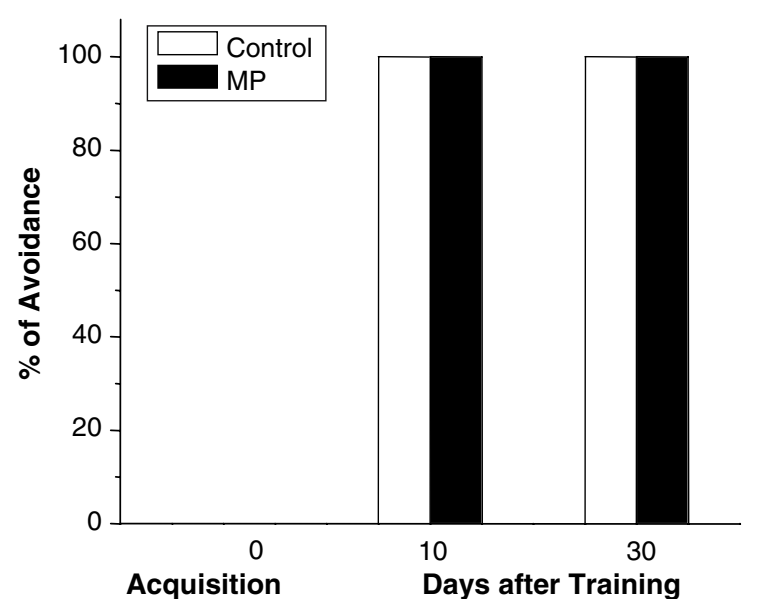

Figure 4. Retention of active avoidance response obtained at post-training day 10 and 30 in control $(n=7)$ and MP-withdrawal rats (MP, $n=8)$. Details for the test were described in 'Materials and methods'.

uous decline in AChE activity in the brain throughout the 3-week dosing may provide an explanation for the lack of tolerance to the suppression of locomotor activity. In comparison with the results reported in Russell et al. [13] and Bushnell et al. [14], the tolerance of the locomotor effects by repeated DFP (diisopropylfluorophosphate) administration showed that the differences in the rate of $\mathrm{AChE}$ inhibition existing between AChE inhibitors are likely to contribute to the different patterns of motor deficits.

The fact that the behavioral deficits in motor function developed slowly and did not fade as did peripheral hyper-cholinergic signs suggests the involvement of the long-lasting functional effects in the central nervous system. Since the most dramatic neurochemical changes were found in the striatum (Table 1) and in view of the significance of this area in regulation of motor functions, we hypothesize that the motor dysfunction upon methyl parathion exposure is associated with an over-stimulation on dopaminergic neuron in basal ganglia. It has been demonstrated that pedunculopontine cholinergic neurons which innervate the substantia nigra pars compacta, modulate motor behavior by increasing the activity of dopaminergic nigrostriatal pathway [15]. Anticholinergic drugs such as atropine are known to increase locomotor activity. Conversely, injection of the cholinergic agonist, carbachol, into the basal forebrain decreased locomotor activity. Significant 
Table 1. Time schedule for different behavioral tests performed and corresponding neurochemical changes during the dosing (neurochemical data are adapted from [12]).

\begin{tabular}{|c|c|c|c|c|c|}
\hline & \multicolumn{3}{|c|}{ Neurochemical studies } & \multicolumn{2}{|c|}{ Neurobehavioral studies } \\
\hline & $\mathrm{AChE}(\%)$ & $\mathrm{M}_{1}(\%)$ & $\mathrm{M}_{2}(\%)$ & Locomotion & Active avoidance \\
\hline Pre-injection (Day 0) & 100 & 100 & 100 & Yes & \\
\hline Day 7 & & & & $\Delta$ & \\
\hline Frontal cortex & 34 & 101 & 91 & & \\
\hline Hippocampus & 46 & 105 & 95 & & \\
\hline Striatum & 37 & 98 & 77 & & \\
\hline Thalamus & 33 & - & 94 & & \\
\hline Midbrain & 26 & - & 94 & & \\
\hline Day 21 & & & & Yes & \\
\hline Frontal cortex & 13 & 78 & 78 & & \\
\hline Hippocampus & 20 & 91 & 89 & & \\
\hline Striatum & 20 & 78 & 62 & & \\
\hline Thalamus & 13 & - & 94 & & \\
\hline Midbrain & 15 & - & 89 & & \\
\hline
\end{tabular}

Post-injection

reductions in locomotion were also observed after depletions of dopamine in the medial striatum and the nucleus accumbens [16]. Earlier studies in this lab already addressed the involvement of other neurotransmitter systems, dopaminergic and GABAergic system) in OP antiAChE-induced neurotoxicity. We reported that the changes in dopaminergic system arose secondarily to an elevation of brain acetylcholine following cholinesterase inhibition [17, 18]. Chronic administration of DFP reduced the number of muscarinic sites, but increased dopamine and GABA receptor densities [17]. Further, a decrease in dopamine level at striatum was detected after both acute and chronic exposure to DFP [18]. Together with our present observations, we suspect that OP antiAChE-caused hyper-cholinergic stimulation may disrupt the balance between cholinergic and dopaminergic system, resulting in decreases in motor activity. To clarify the role of dopaminergic system in MP-induced behavioral changes at motor activity, further investigation is needed.

In our study, rats exposed to MP prior to active-avoidance training were not impaired in the ability of acquisition or retention of the task. Our data did not support the hypothesis that repeated MP exposure leads to the impairment of learning and memory behavior. The data regarding the cognitive effects of OP pesticides had been contradictory since 1960 s. So far there were several lines of evidence consistent with our findings. For example, Costa and Murphy [19] reported the retention of a single trial passive avoidance task was not impaired in mice tolerant to disulfoton. In their experiment, the number of muscarinic receptors was decreased $39 \%$ in the striatum and $20 \%$ in the hippocampus, which was quite similar to the receptor levels in our study. Llorens et al. [20] also reported disulfoton did not impair passive avoidance acquisition or retention, but did impair the acquisition of water maze task. However, other groups have reported that passive avoidance responding was impaired when rats were trained after a series of injections of DFP [21]. Upchurch and Wehner [22] observed impaired acquisition, retention, and reversal of spatial learning in mice which had been dosed repeatedly with DFP before training; mice trained before dosing were not affected. The use of different OP antiAChEs, routes of administration, dosage and duration of treatment, especially the different behavioral tasks chosen to evaluate the performance of learning and memory makes it difficult to form a consistent view on the cognitive effects of OP exposure.

In a series of studies done by Bushnell et al. [14, $23,24]$ on behavioral and neurochemical effects of 
DFP and chlorpyrifos, they concluded that deficits induced by repeated exposure to OP antiAChE could not be attributed entirely to the inhibition of $\mathrm{AChE}$ and down-regulation of muscarinic receptors. Later studies on low-level repeated exposure to chlorpyrifos revealed the impairment of cognitive function in the absence of significant brain $\mathrm{ChE}$ inhibition and reduction in muscarinic receptor [25]. The lack of correlation between the neurochemical changes and behavioral effects upon repeated exposure to OPs makes us to question that whether AChE activity and muscarinic receptor reduction are reliable biomakers for the assessment of the health risk of long-term toxicity of OPs. The different functional deficits between compounds may result from the actions of OP other than their ability to inhibit AChE. Such processes may include neural damage induced by chronic exposure to OP antiAChE [26] or alteration in receptor transduction [27]. Pope [28] evaluated the comparative toxicity of $38 \mathrm{OP} \mathrm{AChE}$ inhibitors currently registered for use as pesticides in the United States and suggested that some OP pesticides have toxicologically relevant sites of action in addition to AChE. These additional actions may contribute to qualitative and quantitative differences in toxicity by different OP pesticides. The inhibition of cholinesterase is a reliable endpoint for the assessment of the acute toxicity of OPs, but may not be suitable for the long-term toxicity. Further investigations to find those additional sites of action and to correlate with the behavioral responses are very important for accurate assessment of chronic toxicity of OP pesticides.

Here, we investigated the possible adverse effects of repeated exposure to MP. MP is a highly toxic compound in terms of acute toxicity compared to DFP or chlorpyrifos, which have received much more attention and widely studied. Our study revealed a suppressant effect on motor activities in MP-treated rats. But no impairment in acquisition or retention of an active avoidance response was found in the current study. However, we do not exclude the possibility that impairments on cognitive function could be detected at a longer exposure or by other tasks. Our data demonstrated that repeated exposure to MP caused some functional deficits in CNS, but motor activity and associative learning/memory process may differ in the sensitivity to the toxic effect. OP pesticide with highly acute toxicity does not necessarily cause severe adverse effects in a longer exposure at sublethal doses. The behavioral effects upon chronic exposure to MP and possibly other OP compounds cannot be attributed entirely to brain AChE inhibition. This inconsistency strongly urges the need to identify novel biomarkers for assessment of long-term neurotoxicity of OPs.

\section{References}

1. Karczmar A.G., Acute and long lasting central actions of organophosphorus agents. Fundam. Appl. Toxicol. 4: S1S17, 1984.

2. Hoskins B. and Ho I.K., Tolerance to organophosphorus cholinesterase insecticides, In: Chambers J.E. and Levis P.E. (Eds.), Organophosphates: Chemistry, Fate, and Effects. Academic Press, San Diego, 1992, pp. 285-297.

3. Russell R.W. and Overstreet D.H., Mechanisms underlying sensitivity to organophosphorus anticholinesterase compounds. Prog. Neurobiol. 28: 97-129, 1987.

4. Gershon S. and Shaw F.H., Psychiatric sequelae of chronic exposure to organophosphorus insecticides. Lancet 11: 1371-1374, 1961.

5. Metcalf D.R. and Holmes J.H., EEG, psychological and neurological alterations in humans with organophosphorus exposure. Ann. N.Y. Acad. Sci. 160: 357-365, 1969.

6. Le Couteur D.G., McLean A.J., Taylor M.C., Woodham B.L. and Board P.G., Pesticides and Parkinson's disease. Biomed. Pharmacother. 53: 122-130, 1999.

7. Carriero D.L., Outslay G., Mayorga A.J., Aberman J., Gianutsos G. and Salamone J.D., Motor dysfunction produced by tacrine administration in rats. Pharmacol. Biochem. Behav. 58: 851-858, 1997.

8. Ott B.R. and Lannon M.C., Exacerbation of Parkinsonism by tacrine. Clin. Neuropharmacol. 15: 322-325, 1992.

9. United States Environmental Protections Agency. Illegal indoor use of methyl parathion. Retrieved from http:// www.epa.gov/pesticides/citizens/methyl.htm, 2000.

10. Agency for Toxic Substances and Disease Registry. Illegal use of methyl parathion insecticide. Retrieved from http:// www.atsdr.cdc.gov/alerts/961213.htm, 1996.

11. Kramer R.E., Wellman S.E., Rockhold R.W. and Baker R.C., Pharmacokinetics methyl parathion: a comparison following single intravenous, oral or dermal administration. J. Biomed. Sci. 9: 311-320, 2002.

12. Sun T.T., Ma T.G. and Ho I.K., Differential modulation of muscarinic receptors in the rat brain by repeated exposure to methyl parathion. J. Toxicol. Sci. 28: 427-438, 2003.

13. Russell R.W., Booth R.A., Smith C.A., Jenden D.J., Roch M., Rice K.M. and Lauretz S.D., Roles of neurotransmitter receptors in behavior: recovery of function following decreases in muscarinic receptor density induced by cholinesterase inhibition. Behav. Neurosci. 103: 881-892, 1989.

14. Bushnell P.J., Padilla S.S., Ward T., Pope C.N. and Olszyk V.B., Behavioral and neurochemical changes in rats dosed repeatedly with diisopropylfluorophosphate. J. Pharmacol. Exp. Ther. 256: 741-750, 1991

15. Gongora-Alfaro J.L., Hernandez-Lopez S., Martinez-Fong D., Flores G. and Aceves J., Circling behavior elicited by cholinergic transmission in the substantia nigra pars 
compacta: involvement of nicotinic and muscarinic receptors. Neuroscience 71: 729-734, 1996.

16. Cousins M.S., Sokolowski J.D. and Salamone J.D., Different effects of nucleus accumbens and ventrolateral striatal dopamine depletions on instrumental response selection in the rat. Pharmacol. Biochem. Behav. 46: 943951, 1993.

17. Sivam S.P., Norris J.C., Lim D.K., Hoskins B. and Ho I.K., Effects of acute and chronic cholinesterase inhibitor with diisopropylfluorophosphate on muscarinic, dopamine and GABA receptors of the rat striatum. J. Neurochem. 40: 1414-1422, 1983.

18. Fernando J.C., Hoskins B. and Ho I.K., Effect on striatal dopamine metabolism and differential motor behavior tolerance following chronic cholinesterase inhibition with diisopropylfluorophosphate. Pharmacol. Biochem. Behav. 20: 951-957, 1984.

19. Costa L.G. and Murphy S.D., Passive avoidance retention in mice tolerant to the organophosphorus insecticide disulfoton. Toxicol. Appl. Pharmacol. 65: 451-458, 1982.

20. Llorens J., Crofton K.M., Tilson H.A., Ali S.F. and Mundy W.R., Characterization of disulfoton-induced behavioral and neurochemical effects following repeated exposure. Fundam. Appl. Toxicol. 20: 163-169, 1993.

21. Gardner R., Ray R., Frankenheim J., Wallace K., Loss M and Robichaud R., A possible mechanism for diisopropylffurophosphate-induced memory loss in rats. Pharmacol. Biochem. Behav. 21: 43-46, 1984.
22. Upchurch M. and Wehner J.M., Effects of chronic diisopropylfluorophosphate treatment on spatial learning in mice. Pharmacol. Biochem. Behav. 27: 143-151, 1987.

23. Bushnell P.J., Kelly K.L. and Ward T.R., Repeated inhibition of cholinesterase by chlorpyrifos in rats: behavioral, neurochemical and pharmacological indices of tolerance. J. Pharmacol. Exp. Ther. 270: 15-25, 1994.

24. Bushnell P.J., Pope C.N. and Padilla S., Behavioral and neurochemical effects of acute chlorpyrifos in rats: tolerance to prolonged inhibition of cholinesterase. J. Pharmacol. Exp. Ther. 266: 1007-1017, 1993.

25. Jett D.A., Navoa R.V., Beckles R.A. and McLemore G.L., Cognitive function and cholinergic neurochemistry in weanling rats exposed to chlorpyrifos. Toxicol. Appl. Pharmacol. 174: 89-98, 2001.

26. Gupta R.C., Patterson G.T. and Dettbarn W.D., Mechanisms of toxicity and tolerance to diisopropylphosphorofluoridate at the neuromuscular junction of the rat. Toxicol. Appl. Pharmacol. 84: 541-550, 1986.

27. Klein W.L., Sullivan J., Skorupa A. and Aguilar J.S., Plasticity of neuronal receptors. Fed. Am. Soc. Exp. Biol. J. 3: 2132-2140, 1989.

28. Pope C.N., Organophosphorus pesticides: do they all have the same mechanism of toxicity? J. Toxicol. Environ. Health B Crit. Rev. 2: 161-181, 1999. 